\title{
Heart Diseases Diagnosis Using Neural Networks Arbitration
}

\author{
${ }^{1}$ Ebenezer Obaloluwa Olaniyi and ${ }^{2}$ Oyebade Kayode Oyedotun \\ ${ }^{1,2}$ Near East University, Lefkosa, via Mersin 10, Turkey, Department of Electrical/Electronic Engineering, Member \\ Center of Innovation for Artificial Intelligence \\ Email: obalolu117@yahoo.com;oyebade.oyedotun@yahoo.com
}

\author{
${ }^{3}$ Khashman Adnan \\ ${ }^{3}$ Founder (and Director), Center of Innovation for Artificial Intelligence British University of Nicosia, Via Mersin 10, \\ Turkey \\ Email: adnan.khashman@bun.edu.tr
}

\begin{abstract}
There is an increase in death rate yearly as a result of heart diseases. One of the major factors that cause this increase is misdiagnoses on the part of medical doctors or ignorance on the part of the patient. Heart diseases can be described as any kind of disorder that affects the heart. In this research work, causes of heart diseases, the complications and the remedies for the diseases have been considered. An intelligent system which can diagnose heart diseases has been implemented. This system will prevent misdiagnosis which is the major error that may occur by medical doctors. The dataset of statlog heart disease has been used to carry out this experiment. The dataset comprises attributes of patients diagnosed for heart diseases. The diagnosis was used to confirm whether heart disease is present or absent in the patient. The datasets were obtained from the UCI Machine Learning. This dataset was divided into training, validation set and testing set, to be fed into the network. The intelligent system was modeled on feed forward multilayer perceptron, and support vector machine. The recognition rate obtained from these models were later compared to ascertain the best model for the intelligent system due to its significance in medical field. The results obtained are $85 \%, 87.5 \%$ for feedforward multilayer perceptron, and support vector machine respectively. From this experiment we discovered that support vector machine is the best network for the diagnosis of heart disease.
\end{abstract}

Index Terms-Diagnosis, Heart disease, Neural network, Support vector machine.

\section{INTRODUCTION}

The heart can be described as the size of a fist and a strong muscle in the body. The heart beats to pump blood around the body, by pushing it from left chambers through arteries of ever decreasing size and finally reaching the capillaries in all the parts of the body [1] Whenever the body has finished taking oxygen and nutrients from the blood, the blood returned to the heart through the veins in the right chamber of the heart, on its way back, the blood passes through the liver and waste product are removed.

Any disorderliness that affects the heart from infection to genetic defects and blood vessel disease is referred to as heart disease [2]. Heart disease is a serious disease and its diagnosis at early stage remains challenging task [3]. Many people are unaware on time that they have heart disease as a result of improper check up of lipid profile which measure amount of triglycerides and either good and bad cholesterol in the blood. In fact, up to $25 \%$ of people with heart disease have no symptoms despite insufficient blood flow to the heart, a condition that is referred to as silent heart disease [4]. This has led to several complications such as heart failure which is when the heart is no longer able to pump enough blood that the body needs to function well. Heart attack is the complete blockage of a coronary artery which interrupts blood flow to a portion of the heart muscle causing death of the heart tissue. Also, it has led to stroke which occur as a result of blood clots that form in the blood vessels that lodge in blood into the brain. The other complication is death, which occurs as a result of not given proper attention to the heart disease or unawareness on time as a result of silent symptoms.

Heart disease is a leading cause of death for both man and woman throughout the world. In the United State of America about 600,000 people die as a result of heart disease every year which is calculated to be one in every four deaths [5]. The rate of death caused by heart disease among races in America in the year 2008 is illustrated in the table below.

Table 1. Death Rate Among Races In America [5]

\begin{tabular}{|l|l|}
\hline Races & Death Rate \\
\hline White America & $25.1 \%$ \\
\hline African American & $24.5 \%$ \\
\hline Asian or Pacific Islander & $23.2 \%$ \\
\hline Hispanic Community & $20.8 \%$ \\
\hline American Indian and Alaska Native & $18 \%$ \\
\hline
\end{tabular}


In this research work, we have used the heart disease dataset obtained from the UCI Machine Learning to develop intelligent systems for diagnosis of heart disease. The results obtained from these systems were compared and the highest recognition rate obtained was taken as the best system for diagnosis of heart disease. This system will solve the problem of misdiagnose of heart disease.

The remaining part of the paper is arranged as follows: section 2 is the related works on the heart disease and explanation on the dataset used to carry out the experiment, section 3 is the model section which contains all explanation on working principles of the models used. Section 4 is the performance evaluation, section 5 is the conclusion.

\section{RELATED WORK}

Milan Kumari et al. [6], [7] used data mining classification techniques RIPPER classifier, Decision Tree, Artificial Neural Networks and Support Vector Machine to analyse Cardiovascular disease dataset. He later compared the performance of these techniques through sensitivity, Specificity, Accuracy, Error Rate, True Positive Rate, and False Positive Rate. He made used of 10-fold cross-validation method to measure the unbiased estimation of these prediction models. The result obtained for RIPPER, Decision Tree, ANN, and SVM are 0.2756, 0.2755, 0.2248, and 0.1588 respectively. The accuracy of the RIPPER, Decision Tree, ANN, and SVM are $81.08 \%, 79.05 \%, 80.6 \%$, and $84.12 \%$ respectively.

Resul Das and Ibrahim Turkoglu [8] used a methodology which SAS software was introduced for diagnosing of heart disease. He placed a neural network ensemble method at the centre of the proposed system. Then this ensemble based method creates new models by combining the posterior probabilities or predicted values from multiple predecessor models. This results in creation of more effective models. The experiment was performed with the proposed tool and $89.01 \%$ classification accuracy was obtained from the experiments made on the data obtained from the Cleveland heart disease database. The sensitivity and specificity value of $80.95 \%$ and $95.91 \%$ were obtained for the heart diagnosis.

Niti Guru et al. [9] used the Neural network to predict heart disease, blood pressure, and sugar. He performed a set of experiments on a sample of a database of 78 patient records, 13 input variables (Age, Blood Pressure, Angiography's report etc.) are used to train and test the neural network. He suggested supervised network for the diagnosis of heart disease and trained it using backpropagation algorithm. The system was trained for 78 patients' records. On the trained data, when unknown data are entered by Doctor, the system will find the unknown data from the trained data and generate lists of possible disease from which patients can suffer. He discovered that human error can be avoided in this system. Therefore the system is more reliable and helps doctors to take control decision.
Hongmei [10], [1] employed a computational model based on a multilayer perceptron (MLP) neural network with three layers to develop a support system for diagnosis of five major heart disease. He used 38 input attributes that were extracted from a large number of patient cases. He determined the number of hidden layer neurons through the cascade learning process. He had five output neurons where each stands for the heart disease of interest. The author trained the network using backpropagation algorithm augmented with momentum rate, adaptive learning rate and forgetting mechanics. Besides, He handled the missing data by applying substituting mean method. From his experiment, it was discovered that adopted MLP-based decision model can achieve a high accuracy level 63.2 - 82.9\% on classification of heart disease, qualifying it as a good decision support system deployable for clinical diagnosis.

John Gennari proposed CLASSIT Conceptual clustering system using Cleveland database and achieved an accuracy of $78.9 \%$ [11].

Detrano et.al [12] used a logistic regression derived discriminant function and achieved a correct classification accuracy of approximately $77 \%$

Rajkumar and Sophia proposed diagnosis of heart disease using data mining algorithm. The diagnosis of a heart attack is initially made by combining a chemical symptoms and a characteristic electrocardiogram (ECG) changes. From this research, an accuracy of $53.33 \%$ was obtained [13], [14].

To figure axis labels, use words rather than symbols. Do not label axes only with units. Do not label axes with a ratio of quantities and units. Figure labels should be legible, about 9-point type.

Color figures will be appearing only in online publication. All figures will be black and white graphs in print publication.

\section{A. Database}

This dataset was taken from the UCI machine learning repository. The heart disease dataset is made up of 75 raw attributes from which 13 attributes were published [15]. These attributes are very vital in the diagnosis of heart disease. The attribute include fasting blood sugar test which must indicate $\prec 120 \mathrm{mg} / \mathrm{dl}$ for a patient with absent test result and present test result of $\succ 120 \mathrm{mg} / \mathrm{dl}$ for a patient that has heart disease. Also, a patient that has serum cholestorol greater than $180 \mathrm{mg} / \mathrm{dl}$ is also considered as heart disease present.

The 13 attributes considered in this research work are stated below [15]:

Attribute 1: Age

Attribute2: Sex

Attribute3: Chest pain type 4values

Attribute4: Resting blood pressure

Attribute5: Serum cholesterol in $\mathrm{mg} / \mathrm{dl}$

Attribute6: Fasting blood sugar $\succ 120 \mathrm{mg} / \mathrm{dl}$

Attribute7: Resting electrocardiographic result (value 0, $1,2)$ 
Attribute8: Maximum heart rate achieved

Attribute9: Exercise induced angina

Attribute10: Old peak $=$ ST depression induced by exercise relative to the rest

Attribute11: The slope of the peak exercise ST segment

Attribute12: Number of major vessels $(0-3)$ coloured by flourosopy

Attribute13: Thal: $3=$ normal; $6=$ fixed defect; $7=$ reversible defect.

\section{B. Normalization of Attributes}

In order for the classifier to perform better, there is a need for transforming the feature values into homogenous and well behaved values that yield numerical stability [16], [17]. This is done by determining the highest value of each attribute. This determined value of each attribute will then be used to divide each feature of its own attributes. This done to achieve a value between 0 and 1 in each attribute. This will enhance the training of the network to give the best classifier.

The following are the highest number obtained from each attribute of the heart disease dataset.

Table 2. The Maximum Sample Value

\begin{tabular}{|l|l|}
\hline Attributes & Highest Value \\
\hline Age & 77 \\
\hline Sex & 1 \\
\hline Chest Pain Type & 4 \\
\hline Resting Blood Pressure & 200 \\
\hline Serum Cholesterol(mg/dl) & 564 \\
\hline Fasting Blood Sugar & 1 \\
\hline Resting Electrocardiographic result & 2 \\
\hline Maximum Heart Rate Achieved & 202 \\
\hline Exercise Induced Angina & 1 \\
\hline Old Peak & 6.2 \\
\hline $\begin{array}{l}\text { The Slope of the Peak Exercise ST } \\
\text { Segment }\end{array}$ & 3 \\
\hline Number of Major Vessels & 3 \\
\hline Thal; fixed defect; reversible defect & 7 \\
\hline
\end{tabular}

From the table above, we denoted the sex (male and female) using 1 for man and 0 for woman, so that we have data that is useful in the research work.

Each highest value of each corresponding attribute is used to divide each other in order to obtain a value that ranges from 0 to 1 .

$$
N . V .=\frac{\text { Each Attributes Samples }}{\text { Corresponding Maximum Values }}
$$

Where N.V is the normalized value of the dataset

The following values are obtained from the normalization of samples. The table below illustrates the first four attributes with its first five corresponding normalized sample values.

Table 3. Normalized Samples

\begin{tabular}{|c|c|c|c|c|c|}
\hline Attributes & \multicolumn{5}{|c|}{ Samples } \\
\hline Age & 0.909 & 0.870 & 0.7403 & 0.8312 & 0.961 \\
\hline Sex & 1 & 0 & 1 & 1 & 0 \\
\hline $\begin{array}{c}\text { Chest Pain } \\
\text { Resting }\end{array}$ & 1 & 0.7550 & 0.5 & 1 & 0.5 \\
\hline $\begin{array}{c}\text { Blood } \\
\text { Pressure }\end{array}$ & 0.650 & 0.575 & 0.62 & 0.64 & 0.6 \\
\hline
\end{tabular}

\section{Artificial Neural Networks}

Artificial Neural Network is the mimicking of human neuron on a system. This is done to develop a system that can perform the activities of the human brain. Artificial neural network is a multilayer network made up of input layer neurons, hidden neuron and output neurons.[18][19] The neural network can also be described as a collection of the activatable unit (neurons) in which connections are weighted, usually with real-value weights [20].

In experimenting artificial neural network, it required three parts, the first part is the training and the second part is the validation part and the third part is the testing part, the training data is used to train or teach the network a complex problem that it want to solve, the validation data are employed to stop the neural network during training, when its moving toward overfitting. It is used to prevent overfitting in neural network and the test data are employed in the network to assess the performance of the network, if the network has really understood the pattern that it's learned or not. There are many algorithms that have been used in artificial Neural Networks. One of examples of such algorithm is multilayer perceptron. Multilayer perceptron trained with the backpropagation network by updating the synaptic weight and test by feedforward network. The Fig. 1 below is a typical representation of feedforward neural networks.

Backpropagation neural network is a supervised learning and its learn by example. It is the most well known neural network because it is simple to understand and very easy to apply. Backpropagation neural network is a learning technique that adjusts its weight by propagating its weight changes from the sink to the source [21]. The optimal goal of this learning system is to have a minimal error which is relative to having outputs which is the same as or closer to the target.

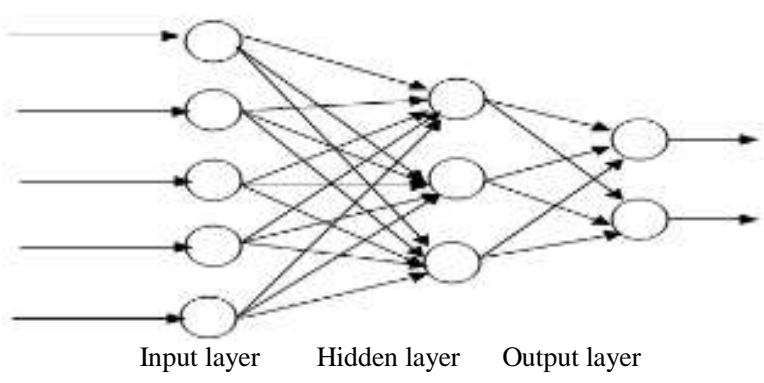

Fig.1. Typical Neural Network Topology. 
Backpropagation is made up of three layers which are input layer, hidden layer and output layer. During propagation, the patterns to be learned by the network are input to the network through the neurons in the input layer. The neurons in the input layer are determined by the number of attributes or application required in the experiment. The output from the input layer is passed through the synaptic weight to the hidden layer. The hidden layer is a processed layer which made up of sum function and activation function. The activation function used in this research is sigmoid function due to it's soft switching operation.

$$
f(x)=1 / 1+e^{-x}
$$

In this hidden layer, the product of the input pattern from the input layer with the weight between the input layer and the hidden layer are summed and activation function is acted on it, the result produced determined by the threshold value of the activated function. Then another synaptic weight between the hidden layer and the output layer passed the output of the hidden layer to the output layer. The output layer is also a processing unit which comprises summation function and activation function. In the output layer, the summation function summed up the product of the activated output of the hidden layer and the synaptic weight between the hidden layer and output layer and passed it to the activation function to decide the final output using the threshold value of the activation function.

When the output of the network is produced, this will be compared to the target then an error is formed.

$$
\text { Error }=\text { desired output }- \text { Actual Output }
$$

This error then sent backward to the input from the output by updating the synaptic weight. This process is repeated until the minimum error is achieved. Learning rate and momentum rate are introduced for gradient descent to find the weight that minimizes error. The following are the equations of backpropagation in the output layer and the hidden layer [16].

Output layer

The error is calculated by comparing the target with the actual output.

$$
\begin{gathered}
\Delta_{z}=d_{z}-O_{z} \\
\delta_{z}=\Delta_{z} O_{z}\left(1-O_{z}\right) \\
\delta_{z}=\left(d_{z}-O_{z}\right) O_{z}\left(1-O_{z}\right)
\end{gathered}
$$

Where

$O_{z}=$ the activation value

$d_{z}=$ Desired output neuron value

$\mathrm{Oz}(1-\mathrm{Oz})=$ derivative of a sigmoid function

Hidden layer
The following equations are used to update the synaptic weight between the output layer and the hidden layer

$$
\begin{gathered}
\Delta W_{y_{u} z}=\eta \delta_{z} X_{z} \\
W_{y_{u} z}=W_{y_{z} z}+\Delta W_{y_{z} z}
\end{gathered}
$$

$\Delta W_{y_{z} z}=$ the change in synaptic weight between hidden layer and output layer.

$\eta=$ learning rate, which is a relatively small constant that indicate the relative change in synaptic weights. It defines the learning power of the neural network.

To enhance the process of changes in the synaptic weights a modification to equation 5 is given below:

$$
\Delta W_{y_{z}}^{m}=\eta \Delta W_{y_{z}}^{m-1}
$$

The learning rate is the learning power of the neural network and the momentum rate defines the speed at which the neural network learns. It also helps to prevent the neural network from settling down at the local minima.

\section{Support Vector}

Support vector machine is a novel supervised machine learning techniques, proven to be particularly attractive to biological analysis due to their ability to handle noise and large input spaces [22]. Unlike perceptron algorithm that find any linear separator or Naive Bayes that look for the best linear separator with the aid of some criterion. Support Vector Machine in particular defines the criterion to be looking for a decision surface that is maximally far away from any data point [23]. The margin of the classifier is determined by the distance from the decision surface to the closest pattern point. This means that the decision function of pattern is usually specified by the subset of the pattern or small portion of pattern that specify the position of the classifier. The small portion of the pattern or a subset of pattern refers to as support vector.

Support vector machine can be described as representative of samples as a point in space mapped so that the samples of different categories are separated by the clear gap that has wide possibility [24]. The main goal in support vector machine is to have a maximum margin between between different samples and keep the margin separated. For instance, given a set of point that belong to two classes, Support Vector Machine find the hyperplane with the largest fraction of the pattern of the same class on the same plane. This is referred to as the optimal hyperplane. The use of support vector machine minimizes the classification problem or misdiagnosis. form

Assuming label training data as a data point of the

$$
\mathrm{S}=\left(x_{1}, y_{1}\right)\left(x_{2}, y_{2}\right) \ldots \ldots \ldots \ldots\left(x_{n}, y_{n}\right)
$$


Where $y_{n}=+1$ or -1 which is the target that indicate the class to which the point $x_{n}$ belong, $\mathrm{n}$ is the number of data samples.

Support vector machine works in a way that it first map the input vectors into the decision value and then perform the classification using appropriate threshold value. To view the training data, the hyperplane was separated which can be described as mapping

$$
w^{T} x+b=0
$$

Where $\mathrm{w}=$ weight vector of $\mathrm{P}$-dimensional, $\mathrm{b}=$ scalar

The weight vector $\mathrm{w}$ is perpendicular to the separating hyperplane, then the offset parameter $b$ allows to increase or decrease the margin. If the training data are linearly separable, the hyperplane will be selected so that there will be no points between them and then maximizing the distance between the hyperplane. We found out the distance to be $2 / / w \mid$. In order to minimize $/ w /$ we need to ensure that for all (i) either

$$
w x_{i}-b \geq 1 \text { or } w \cdot x-b \leq-1
$$

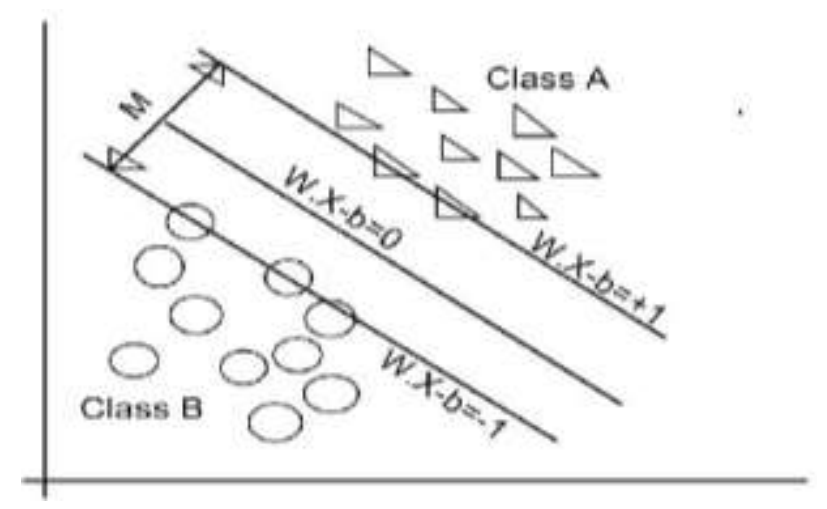

Fig.1. Support Vector Machine

\section{Design Of THE Network}

In this research work, the 270samples are divided into two parts which are the training data and the testing dataset. This division is based on 60: 40, that is, we have 162 training dataset and 108 testing dataset for the network input. The target of the network is coded as $(0$ $1)$, if there is present of heart disease and (1 0 ) if heart disease is absent.

In the backpropagation neural network design, the training dataset, the testing set and the target are converted into a matrix in order to be fed to the network. We obtained 13 x 162 matrix training set with its corresponding target which is $2 \times 162$ matrix and the testing set of $13 \times 108$ matrix with its corresponding target of $2 \times 108$ matrix.

The input layer of the network is made up of 13 neurons, which is equivalent to the number of attributes we considered in this research work. The number of neurons in the hidden layer was experimenting to determine the best number of neurons that will be able to learn the pattern in the data and give the best classification. In this research work, we obtained the best number of hidden neuron experiment from two neurons until we reach six neurons which gave us the best result. There are two functions that occur in the hidden neurons, these are the summation of the synaptic weight and the activation function. The activation function used in this research work is the sigmoid transfer function because of its soft switching and ability to solve complex problems. At the output layer, two neurons were applied, which indicate the target of the network. They represent if the heart disease is present or absent in a patient.

The support machine is designed in a way that it has the input to be a $13 \times 162$ matrix, but the target in SVM is in one dimension. It is represented as either +1 or -1 which gave a matrix of $1 \times 162$. The +1 indicates that the heart disease present and -1 indicate that heart disease absent.

\section{PERformance EVAluation}

The results obtained from this research work are in two phases, the first phase is obtained from the backpropagation training and testing of the network while the second phase is obtained from the support vector machine.

In the backprogation neural network, learning and momentum rates were introduced. The learning rate determines the speed at which the network learns the pattern, if the learning rate is too small. It means that it will take a longer time before the network can learn and on the other hand, if the network learning rate is too high, it makes the network to continually jump over the optimum weights and the network fails to learn. Also, the momentum rate prevents the network from settling down at the local minima. Therefore, the two parameters are experimenting to obtain the best value. The learning rate and momentum rate used in this research work are 0.32 and 0.73 respectively, with six (6) hidden neurons, the time for the network to learn was $20 \mathrm{sec}$ at 2000 epoch. The table 4 below show the result obtained from the feedforward multilayer perceptron with its corresponding minimum error curve.

Table 4. The Artificial Neural Network trained with backpropagation

\begin{tabular}{|c|c|c|c|}
\hline $\begin{array}{c}\text { No. of Input } \\
\text { Neurons }\end{array}$ & 13 & $\begin{array}{c}\text { No. of Hidden } \\
\text { Neurons }\end{array}$ & 6 \\
\hline $\begin{array}{c}\text { No. of Output } \\
\text { Neurons }\end{array}$ & 2 & Epoch & 2000 \\
\hline Learning Rate & 0.32 & $\begin{array}{c}\text { Momentum } \\
\text { rate }\end{array}$ & 0.73 \\
\hline Performance & 0.199 & $\begin{array}{c}\text { Recognition } \\
\text { rate }\end{array}$ & $85 \%$ \\
\hline
\end{tabular}




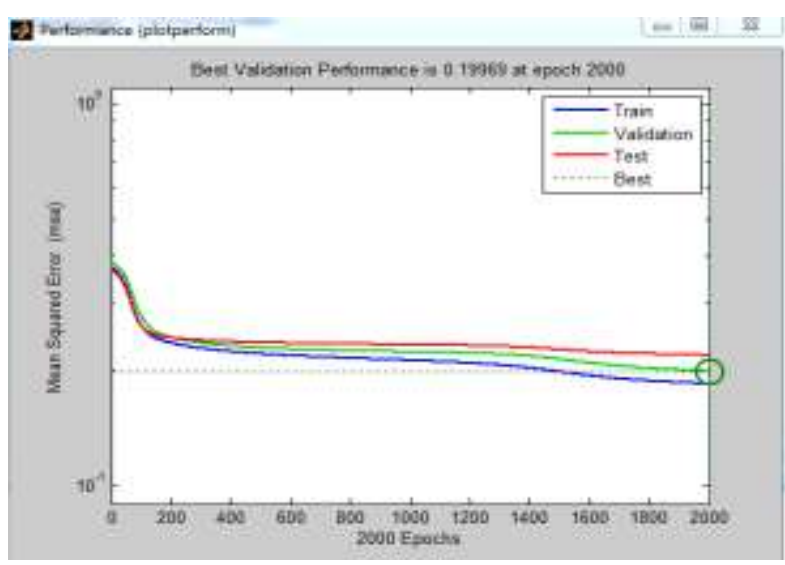

Fig.3. The graph of Mean Squared Error versus Epochs

In the Support vector machine, learning rate and momentum rate were not applicable, it makes use of kernel functions, in this research work linear kernel function was applied and a recognition rate of $87.5 \%$ was obtained from the testing data.

Diagnosis of heart disease can be categorized as thus; when a set of people is examined for presence of heart disease. If some of these people have heart disease and they are diagnosed to be tested positive. This is refers to as true positive (TP). Also, if some of these people do not have the present of heart disease and the test claims negative, this is categorized as true negative (TN). But, in a case where some of the people have heart disease but the test claims that the heart disease is negative. This condition is referred to as false negative $(\mathrm{FN})$. A reverse case can also occur in diagnoses of heart disease. A situation where some of the group of people does not have the heart disease but the test claims that they are positive of the disease. This is categorized as false positive (FP).

Misdiagnosis is a serious offence in medical field. Therefore, care must be taken in diagnosis of patient and optimal performance equipments are required. Due to the misdiagnosis, wrong prescriptions of drugs can be administered to patient, which can lead to death. Therefore, there is a need to consider statistical measure when designing an intelligent diagnosis system for heart disease. The statistical measures that can be considered are sensitivity, specificity, and accuracy. The sensitivity measures the proportion of actual positives which are identified correctly. It diagnoses people living with heart disease correctly as heart disease positive.

$$
\text { Sensitivity }=\frac{T P}{T P+F N} * 100
$$

The specificity measure proportion of people who are correctly identified as not having heart disease, i.e. A condition where a set of healthy people is correctly identified as not having the heart disease.

$$
\text { Specificit } y=\frac{T N}{T N+F P} * 100
$$

In this research work, the specificity, sensitivity and accuracy, were used to determine the performance of the classifier. In addition to selectivity and specificity, the performance can also be measured with positive prediction value (PPV) which determined how well a positive test result actually determines that the disease is present.

$$
P P V=\frac{T P}{T P+F P} * 100
$$

Also, measure the negative prediction value (NPV), which determined how well a negative test result actually determines that the disease is absent.

$$
N P V=\frac{T N}{T P+F N} * 100
$$

Table 5. The Performance Table for Support Vector Machine

\begin{tabular}{|c|c|c|c|}
\hline $\begin{array}{c}\text { No. of Input } \\
\text { Neurons }\end{array}$ & 13 & $\begin{array}{c}\text { No. of Hidden } \\
\text { Neurons }\end{array}$ & N/A \\
\hline $\begin{array}{c}\text { No. of Output } \\
\text { Neurons }\end{array}$ & 1 & Kernel function & Linear \\
\hline True Positive & 38 & True Negative & 53 \\
\hline False Negative & 7 & False Positive & 6 \\
\hline Sensitivity & $84.44 \%$ & Specificity & $89.8 \%$ \\
\hline $\begin{array}{c}\text { Positive } \\
\text { Prediction Value }\end{array}$ & $86.36 \%$ & $\begin{array}{c}\text { Negative } \\
\text { Prediction Value }\end{array}$ & $88.3 \%$ \\
\hline Accuracy & $87.5 \%$ & Epoch & N/A \\
\hline
\end{tabular}

The table 6 below shows the comparison between the two models used to carry out this research. Support vector machine has been discovered to be the best model as a result of the best accuracy obtained from it in this experiment.

Table 1. The Comparison of the Recognition Rate Result of the Experiment

\begin{tabular}{|c|c|}
\hline $\begin{array}{c}\text { Backpropagation Neural Network } \\
\text { (BPNN) }\end{array}$ & $85 \%$ \\
\hline Support Vector Machine & $87.5 \%$ \\
\hline
\end{tabular}

\section{CONCLUSION}

Due to the significance of heart disease diagnosis in the medical field, we have compared two models for diagnosis of this disease. In our result, we discovered that support vector machine is the best algorithm for diagnosis of heart disease which gave a accuracy rate of $87.5 \%$, with high value of sensitivity and specificity. Although, the two results have only difference of $2.5 \%$. Despite, the small difference, support vector machine is recommended as the best algorithm for heart disease diagnosis, which will reduce the chances of misdiagnosis on the part of medical practitioners. 
Besides, in this research work, we have discovered that one of the major causes of death due to heart disease is unawareness at the initial stage of the disease, regular medical checkup can help to discover the disease at initial stage. If heart disease can be discovered in time, it can be properly managed or cured; especially coronary heart disease can be treated by proper diet, medication and exercises.

Finally, the habits that lead to heart disease should also be stopped by an individual to avoid a critical case of the disease. Smoking habit, unhealthy diet and irregular exercises should be stopped.

\section{REFERENCES}

[1] J. S. Sonawane, D. R. Patil and V. S.Thakare, "Survey on Decision Support System for Heart Disease," International Journal of Advancements in Technology, vol 4, pp. 89-96, 2013.

[2] F. C. Pampel, S. Pauley, Progress Against Heart Disease, Praeger Publisher, 2004.

[3] A., Lashsana, R., Noor Ainon R., Zainuddin A., M. Bulgiba "A transparent fuzzy rule-based clinical decision support system for heart disease diagnosis," Knowledge Technology Communications in Computer and Information Science, vol. 295, pp. 62 -71,July, 2011. [online]. Available:

http://link.springer.com/chapter/10.1007\%2F978-3-64232826-8_7.

[4] Dr. Giora Yaron, Symptoms and Complications of Heart_Disease, [online]: ww.itamarmedical.comPatient_Information/Cardio_101/.

[5] M. Bhasin, G. Raghava, "Analysis and Prediction of affinity of TAP bending peptides Using Cascade SVM," Protein Science, vol. 13(3), pp. 596-607, 2004, [online]: http://www.ncbi.nlm.nih.gov/pmc/articles/PMC2286721/.

[6] M. Kumari, S. Godara, "Comparative Study of Data Mining Classification Methods in Cardiovascular Disease Prediction," International Journal of Computer Science and Technology, vol. 2, Issue 2, June (2011).

[7] N. Al-Milli, "Backpropagation neural network for prediction of heart disease," Journal of theoretical and applied information Technology, vol. 56, pp.131-135, Oct 10, 2013.

[8] R. Das, I. Turkoglu, A. Sengur, Effective Diagnosis of Heart Disease through Neural Network Ensemble, "Expert Systems with Applications" vol. 36 issue 4, pp. 76757680, May (2009). [Available]: 10.1016/j.eswa.2008.09.013.

[9] N. Guru, A. Dahiya and N. Rajpal, "Decision Support System for Heart Disease Using Neural Network," Delhi Business Review, vol. 8, No 1, pp. 1 - 6, Jan - June (2007).

[10] S. Prabhat Panday, N. Godara, "Decision Support System for Cardiovascular Heart Disease Diagnosis using Improved Multilayer Perceptron," International Journal of Computer Applications (0975 - 8887) Vol. 45- No.8, May (2012).

[11] Gennari, J. Models of incremental concept formation. Journal of Artificial Intelligence, vol. 1, pp. 11-61., 1989.

[12] Detrano R., et al, International application of a new probability algorithm for the diagnosis of coronary artery disease, American Journal of Cardology, 1989:64(5) 304-310.

[13] A. Rajkumar and G. S. Reena, "Diagnosis of heart disease using data mining algorithm," Global Journal of
Computer Science and Technology, vol. 10, pp. 38-43, December 2010.

[14] Vanisree K., Jythi Singaraju, "Decision Support System for Congenital Heart Disease Diagnosis based on Signs and Symptoms using Neural Networks," International Journal of Computer Applications (0975 - 8887) vol. 19No.6, April 2011.

[15] fftp.ics.uci.edu/pub/machine-learning-databases (last accessed: February 10, 2015).

[16] E. O.Olaniyi, K. Adnan., "Onset Diabetes Diagnosis Using Artificial Neural Network", International Journal of Scientific and Engineering Research, vol. 5, Issue 10, October (2014).

[17] N. Kilic, B. Ekici, S. Hartomacioglu, "Determination of penetration depth at high velocity impact using finite element method and artificial neural network tools," Defence Technology $x x$ pp. 1-13, 2015. [online]: http://dx.doi.org/10.1016/j.dt.2014.12.001.

[18] A. K. Nanila, A. P. Singh, "Fault Diagnosis of MixedSignal Analog Circuit Using Artificial Neural Network", International Journal of Intelligent Systems and Applications, 07, pp. 11-17, 2015. DOI: 10.5815/ijisa.2015.07.02.

[19] S. Bhuvaneswari, J. Sabarathinam, "Defect Analysis Using Artificial Neural Network" I.J. Intelligent Systems and Applications, 05, pp. 33-38, 2013.

[20] M. Melanie, An Introduction to Genetic Algorithms. MIT press, pp. 20-64, 1999.

[21] Magret H. Dunham, data mining Introductory and advanced topics, (2003).

[22] C.H. Ding and I. Dubchak, Multi-class Protein Fold Recognition Using Support Vector Machines and Neural Network, Bioinformatics, vol 17(4) pp. 349-358, April 2001. [online]: http://www.ncbi.nlm.nih.gov/pubmed/11301304.

[23] C. D. Manning, P. Raghavan and H. Schütze, Introduction to Information Retrieval, Cambridge University Press. (2008).

[24] Christopher J.C. Burges, "A Tutorial on Support Vector Machines for Pattern Recognition," Data Mining and Knowledge Discovery, 2, 121-167 (1998).

\section{Authors' Profiles}

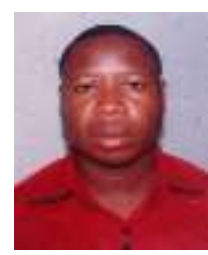

Ebenezer O. Olaniyi, is a member of Centre of Innovation for Artificial Intelligence, British University of Nicosia, Girne, via Mersin-10, Turkey and currently pursuing masters degree program in Electrical/Electronic Engineering at Near East University, Lefkosa, via Mersin-10, Turkey.

His research interest areas are Artificial neural network, Pattern Recognition, Image processing, Machine Learning, Speech Processing and Robotics.

PH- +905428827442. E-mail: obalolu117@yahoo.com

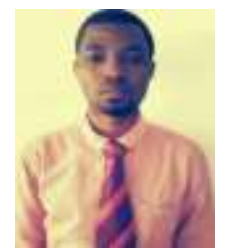

Oyebade K. Oyedotun is a member of Centre of Innovation for Artificial Intelligence, British University of Nicosia, Girne, via Mersin-10, Turkey and currently pursuing masters degree program in Electrical/Electronic Engineering at Near East University, Lefkosa, via Mersin-10, 
Research interests include artificial neural networks, pattern recognition, machine learning, image processing, fuzzy systems and robotics.

PH-+905428892591. E-mail: oyebade.oyedotun@yahoo.com

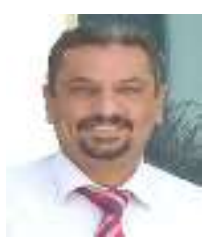

Khashman Adnan received the B.Eng. degree in electronic and communication engineering from University of Birmingham, England, UK, in 1991, and the M.S and Ph.D. degrees in electronic engineering from University of Nottingham, England, UK, in 1992 and 1997.

During 1998-2001 he was an Assistant Professor and the Chairman of Computer Engineering Department, Near East University, Lefkosa, Turkey. During 2001-2009 he was an Associate Professor and Chairman of Electrical and Electronic Engineering Department, and in 2009 he received the professorship. In 2001 he established the Intelligent Systems Research Group (ISRG) at the same university, and has been chairing ISRG until present. From 2007 until 2008 he was also the Vice-Dean of Engineering Faculty, later on from 2013 until 2014 the Dean of the faculty at the same university.

Since 2014, Professor Khashman was appointed as the Founding Dean of Engineering Faculty at the British University of Nicosia in N. Cyprus, where he also established the Centre of Innovation for Artificial Intelligence His current research interests include image processing, pattern recognition, emotional modeling and intelligent systems.

E-mail: adnan.khashman@bun.edu.tr

How to cite this paper: Ebenezer Obaloluwa Olaniyi, Oyebade Kayode Oyedotun, Khashman Adnan,"Heart Diseases Diagnosis Using Neural Networks Arbitration", International Journal of Intelligent Systems and Applications (IJISA), vol.7, no.12, pp.75-82, 2015. DOI: 10.5815/ijisa.2015.12.08 